\title{
Experimental and Theoretical Investigation of the Lamellar Structure of a Styrene-Butyl Methacrylate Diblock Copolymer by Fluorescence Resonance Energy Transfer (FRET), Small-Angle X-Ray Scattering (SAXS) and Self- Consistent Field Simulations
}

\author{
John G. Spiro, Jian Yang, Jian-Xin Zhang ${ }^{\S}$ and Mitchell A. Winnik* \\ Department of Chemistry, University of Toronto, 80 St. George St., Toronto, Ontario, Canada \\ M5S 3 H6 \\ Yahya Rharbi \\ Laboratoire de Rhéologie, BP53 - Domaine Universitaire, 38041 Grenoble Cedex 9, France \\ and Department of Chemistry, University of Toronto \\ Jeffrey D. Vavasour and Mark D. Whitmore \\ Department of Physics and Astronomy, University of Manitoba, Winnipeg, Manitoba, Canada \\ Robert Jérôme \\ Center for Education and Research on Macromolecules, University of Liège, Sart-Tilman B6, \\ 4000 Liège, Belgium

\section{Supporting Information}

This material includes calculation of the free energy of mixing for our PS- $b$-PBMA samples, discusses the implications of the results, mentions the possible role of specific interactions for PS- $b$-polymethacrylates, and notes recent experimental and theoretical studies involving PS- $b$ poly(n-pentyl methacrylate) copolymers. It concludes - in agreement with most reviewers of our manuscript - that the conventional (incompressible) Flory-Huggins approach to data analysis is still very useful. 
The phenomenon that polymer liquids which are mutually soluble at low temperature exhibit a liquid-liquid phase transition at higher temperature (lower critical solution temperature - LCST) has been well known for decades; a good discussion was given by McMaster ${ }^{1}$ in 1973 . That a corresponding microphase separation can also take place in block copolymers (lower critical ordering transition - LCOT) was first reported only in 1994, by Russell and coworkers. ${ }^{2}$ In fact, even at that time, PS- $b$-PBMA, the subject of our investigations, was the only copolymer exhibiting this behavior. This demixing - at least in nonpolar systems that lack specific interactions $s^{3,4}$ - is thought to be governed by a delicate balance between enthalpic and noncombinatorial entropic contributions to the free energy of mixing. ${ }^{5.6}$

We have calculated the free energy of mixing of our PS- $b$-PBMA samples $\left(\mathrm{M}_{\mathrm{w}}=\right.$ 180,000, $T=160{ }^{\circ} \mathrm{C}, 50$ volume \% PS) via Ruzette and Mayes' (R\&M) model. ${ }^{6}$ The latter is an attractive formalism, because the input quantities are only pure component properties. Specifically, equations of state (EOS) and solubility parameters (at $25^{\circ} \mathrm{C}$ ) were required for PS and PBMA. The Tait equation for $\mathrm{PS}^{7}$ gave excellent agreement with Quach and Simha's experimental measurements (specific volumes) at approximately $160{ }^{\circ} \mathrm{C}$ and atmospheric pressure, ${ }^{8}$ and it was even in the right functional form for the R\&M model. But for PBMA, the Tait equation published by Rodgers ${ }^{7}$ gave a much lower density at $160.2^{\circ} \mathrm{C}$ and one bar pressure than Olabisi and Simha's experimental result. ${ }^{9}$ Therefore, for PBMA we employed the SanchezLacombe EOS (much more satisfactory), in the form and with parameters given in Reference 10.

Probably the most challenging aspect of using the R\&M model is the solubility parameter $\left(\delta_{25}\right)$ inputs. We do not wish to delve too far into this controversial (particularly for polymers) matter, but will present two calculations, based on the latest (to our knowledge) published values ${ }^{11}$ based on theory as well as on extracting experimental data (such as they are) from Polymer Handbook. ${ }^{12}$ We tend to agree with the authors of Reference 11 that their calculations for $25{ }^{\circ} \mathrm{C}$ are the best available information, especially for comparing two polymers whose glass transition temperatures are very different, such as PS and PBMA. As for the Polymer Handbook data they have listed, $\delta_{25}$ for PBMA seems relatively well established, but for PS there is such a broad range of published "experimental" data ${ }^{12}$ that it is difficult to surmise - nor is it our intention to do so - why Utracki and Simha ${ }^{11}$ selected $\delta_{25}($ experimental $)=18.7 \mathrm{MPa}^{1 / 2}$. 
Be that as it may, the "experimental" $\delta_{25}$ values quoted in Reference 11, combined with the equations of state we used (see above), lead to very reasonable results as far as the phase behavior of symmetrical PS- $b$-PBMA is concerned, with combinatorial entropic (CE), exchange interaction energy (EIE) and EOS contributions

$\Delta_{\mathrm{CE}}=-0.027 \mathrm{~J} / \mathrm{cm}^{3}, \Delta_{\mathrm{EIE}}=0.49 \mathrm{~J} / \mathrm{cm}^{3}$, and $\Delta_{\mathrm{EOS}}=-0.42 \mathrm{~J} / \mathrm{cm}^{3}$ to the free energy of mixing for our samples. We see the "delicate balance, ${ }^{, 5,6}$ of enthalpic and entropic contributions, leading to microphase separation for our molecular weight, but suggesting an LCOT of $160^{\circ} \mathrm{C}$ for 75,000 molecular weight, in reasonable agreement with the experimental results of Russell and coworkers. $^{2}$

However, the picture is very different if we use the theoretical ${ }^{11}$ - and likely more reliable - $\delta_{25}$ values as inputs into the R\&M model. We now obtain $\Delta_{\mathrm{CE}}=-0.027 \mathrm{~J} / \mathrm{cm}^{3}, \Delta_{\mathrm{EIE}}=$ $0.001 \mathrm{~J} / \mathrm{cm}^{3}$, and $\Delta_{\mathrm{EOS}}=0.35 \mathrm{~J} / \mathrm{cm}^{3}$ for our samples. These results do suggest microphases at 160 ${ }^{\circ} \mathrm{C}$, and that our samples would likely be phase separated at any temperature, which again agrees with the experiments ${ }^{2}$; but the results also suggest that there can be no LCOT until we get to very low molecular weights. This not only contradicts the experimental data, but challenges the R\&M model's applicability to PS-b-PBMA.

We conclude that the EOS methods have not matured yet to the stage that they would be useable with any degree of confidence. We have mentioned ${ }^{3,4}$ that there is also a question whether specific interactions, rather than compressibility, are responsible for the experimental observations on PS- $b$-poly(n-methacrylate) copolymers. And in addition, very recent experimental results, obtained by Kim, Russell and coworkers ${ }^{13}$ on PS-b-poly(n-pentyl methacrylate) "revealed an astonishing phase diagram exhibiting an immiscibility loop”, ${ }^{14}$ leading to a need for new theoretical treatments. ${ }^{14}$

Until more progress has been accomplished on incorporating compressibility into PS-bPBMA models, our "incompressible" approach to PS- $b$-PBMA morphology - and hence the $\chi_{\mathrm{FH}}$ ranges and related matters discussed in the main body of our manuscript - remain very much alive. 


\section{REFERENCES AND NOTE}

1 McMaster, L. P. Macromolecules 1973, 6, 760.

2 (a) Russell, T.P.; Karis, T.E.; Gallot, Y.; Mayes, A.M. Nature 1994, 368, 729; (b) Karis, T.E.; Russell, T. P.; Gallot, Y.; Mayes, A.M. Macromolecules 1995, 28, 1129.

3 It is frequently assumed that LCOT is caused by compressibility/equation of state (EOS) effects, but Freed and coworker ${ }^{4}$ could explain the phase behavior of PS-b-poly(n-alkyl methacrylate) diblock copolymers, including PS- $b$-PBMA, in an incompressible treatment incorporating specific interactions of different atom groups.

Dudowicz, J.; Freed, K. F. Macromolecules 2000, 33, 5292.

5 Watkins, J. J.; Brown, G. D.; RamachandraRao, V. S.; Pollard, M. A.; Russell, T. P. Macromolecules 1999, 32, 7737.

6 Ruzette, A-V. G.; Mayes, A. M. Macromolecules 2001, 34, 1894.

7 Rodgers, P. A. J. Appl. Polym. Sci. 1993, 48, 1061.

8 Quach, A.; Simha, R. J. Appl. Phys. 1971, 42, 4592.

9 Olabisi, O.; Simha, R. Macromolecules 1975, 8, 206.

10 Ruzette, A-V. G.; Banerjee, P.; Mayes, A. M.; Pollard, M.; Russell, T. P.; Jérôme,

R.; Slawecki, T.; Hjelm, R.; Thiyagarajan, P. Macromolecules 1998, 31, 8509.

11 Utracki, L. A.; Simha, R. Polym. Int. 2004, 53, 279.

12 Polymer Handbook, $3^{\text {rd }}(1989)$ and $4^{\text {th }}(1999)$ editions.

13 (a) Ryu, D. Y.; Jeong, U.; Kim, J. K.; Russell, T. P. Nature Mater. 2002, 1, 114; (b) Ryu, D. Y.; Jeong, U.; Lee, D. H.; Kim, J.; Youn, H. S.; Kim, J. K. Macromolecules 2003, 36, 2894; (c) Ryu, D. Y.; Lee, D. J.; Kim, J. K.; Lavery, K. A.; Russell, T. P.; Han, Y. S.; Seong. B. S.; Lee, C. H.; Thiyagarajan, P. Phys. Rev. Lett. 2003, 90, 235501; (d) Ryu, D. Y.; Lee, D. H.; Jeong, U.; Yun, S.-H.; Park, S.; Kwon, K.; Sohn, B.-H.; Chang, T.; Kim, J. K.; Russell, T. P. Macromolecules 2004, 37, 3717.

14 Cho, J. Macromolecules 2004, 37, 10101. 